\title{
Long-range electron exchange measured in proteins by quenching of tryptophan phosphorescence
}

(tunneling)

\author{
J. M. Vanderkooi*, S. W. Englander* ${ }^{*}$ S. PapP ${ }^{\dagger}$, W. W. Wright*, and C. S. Owen $\ddagger$ \\ *Department of Biochemistry and Biophysics, School of Medicine, University of Pennsylvania, Philadelphia, PA 19104; ${ }^{\dagger}$ Department of Biophysics, University \\ Medical School of Debrecen, H4012 Debrecen 12, Hungary; and ‡Biochemistry Department, School of Medicine, Jefferson Medical College, \\ Philadelphia, PA 19107
}

Communicated by Mildred Cohn, April 20, 1990

\begin{abstract}
Ten proteins that span a wide range of phosphorescence lifetimes were examined for sensitivity to quenching by four agents of disparate chemical nature. The results show that quenching efficiency is relatively independent of the quencher and is highly correlated with depth of burial of the phosphorescent tryptophan. The bimolecular quenching rate constants $\left(k_{\mathrm{q}}\right)$ measured for the different proteins, spanning 5 orders of magnitude in $k_{\mathrm{q}}$, are found to decrease exponentially with the distance (r) of the tryptophan in angstroms from the protein surface-i.e., $k_{\mathrm{q}}=A \exp (-r / \rho)$, where $A$ is the effective area of the protein. Theoretical analysis shows that this behavior can be expected for an electron-exchange reaction between the buried tryptophans and quenchers in solution in the rapid diffusion limit. Therefore, the results obtained provide evidence for an exponential dependence of electrontransfer rate on distance in a protein environment and evaluate the distance parameter, $\rho$, for electron transfer through the general protein matrix at $1.0 \AA$. For a unimolecular donoracceptor pair with $k_{\mathrm{et}}=k_{\mathrm{o}} \exp (-r / \rho), k_{\mathrm{o}} \approx 10^{9} \mathrm{sec}^{-1}$.
\end{abstract}

In earlier work, we showed that the phenomenon of roomtemperature protein phosphorescence, though previously seen only rarely $(1,2)$, in fact can be found in the great majority of proteins (3). Of 40 proteins surveyed, 29 were found to exhibit phosphorescence in aqueous solution at room temperature with a wide range of lifetimes-between about $0.5 \mathrm{msec}$ and $2 \mathrm{sec}$. The central requirement for the observation of protein phosphorescence in solution is to reduce dissolved oxygen to a sufficiently low level, since oxygen can efficiently quench the excited tryptophan triplet state, even when the tryptophan is buried in the protein matrix (3-6).

A subsequent study (7) revealed that a variety of smallmolecule agents in addition to dioxygen can quench the phosphorescence of protein tryptophans. Surprisingly, even though the phosphorescent tryptophans are well buried within the protein, the quenching efficiency of most of the agents tested (those larger than three atoms in size) was found to be independent of the size and polarity of the quenching agent. This indicates that the quenching process does not involve the penetration of these quenchers through the protein matrix to the position of the buried tryptophan. Although the sensitivity of the different proteins to quenching was found to be spread over a wide range, the various agents tested all quenched any given protein with similar efficiency, indicating that the quenching reaction is determined by some property of the individual tryptophan or the protein itself rather than by the particular quenching agent used. Finally, the quenching rate was essentially independent of solution viscosity. This rules out the possibility of a protein-opening

The publication costs of this article were defrayed in part by page charge payment. This article must therefore be hereby marked "advertisement" in accordance with 18 U.S.C. $\$ 1734$ solely to indicate this fact. reaction that might transiently bring the buried tryptophan into contact with solvent and the added quenchers. All these properties might be explained, it was noted, if the quenching process involves long-range electron transfer occurring on a long time scale, so that the quenchers in solution are effectively in the rapid diffusion limit.

The present work represents an attempt to identify the protein parameters that determine the sensitivity of its tryptophans to the quenching process. We compared various structural parameters of 10 different proteins with their ability to be quenched by four different small-molecule agents. The results show that quenching rate constants decrease exponentially with the distance of the tryptophan from the protein surface, consistent with an electronexchange reaction in the rapid diffusion limit. Analysis of the data then provides an estimate of the dependence of electrontransfer rate on distance when the intervening space is filled with averaged protein matrix.

\section{MATERIALS AND METHODS}

Glyceraldehyde-3-phosphate dehydrogenase (GAPDH) from porcine muscle, Pronase type XIV from Streptomyces griseus, and protease type X from Bacillus thermoproteolyticus rokko were obtained from Sigma. Nuclease from Staphylococcus aureus was a gift of E. E. Lattman (Baltimore). The sources of other proteins and supplies were as listed (7). Protein concentrations were typically $1-2 \mathrm{mg} / \mathrm{ml}$.

Oxygen was removed from the samples, and protein phosphorescence lifetimes were measured as described $(7,8)$. Quenching rate constants were obtained from the dependence of phosphorescence lifetimes on quencher concentration as indicated in Results and ref. 7.

Solution conditions used were as follows: $0.034 \mathrm{M}$ sodium pyrophosphate at $\mathrm{pH} \mathbf{8 . 6}$ for alkaline phosphatase, azurin, aldolase, Pronase, liver alcohol dehydrogenase (LADH), and thermolysin; $0.1 \mathrm{M} \mathrm{NaCl} / 0.01 \mathrm{M}$ sodium phosphate at $\mathrm{pH} 7.0$ for GAPDH and RNase T1; 0.01 M CAPSO [3-(cyclohexylamino)-2-hydroxy-1-propanesulfonic acid] $/ 1 \mathrm{mM} \mathrm{CaCl} 2$ at $\mathrm{pH} 9.2$ for staphylococcal nuclease; and $0.1 \mathrm{M} \mathrm{NaCl} / 1 \mathrm{mM}$ $\mathrm{CaCl}_{2} / 0.01 \mathrm{M}$ Tris at $\mathrm{pH} 7.0$ for parvalbumin. Temperature was $\approx 22^{\circ} \mathrm{C}$.

Coordinates for alkaline phosphatase were obtained from H. W. Wyckoff (New Haven, CT). Other protein coordinates were obtained from the Brookhaven Protein Data Bank (9). Protein structures were analyzed by using the BIOGRAF molecular graphics program (BioDesign, Pasadena, CA) on a microVAX II computer to obtain the distance of the emitting tryptophans from the protein surface. The coordinates of solvent water were added by using the solvation option of BIOGRAF, and structured water molecules inside the protein were excluded. Distances between the tryptophan indole ring

Abbreviations: LADH, liver alcohol dehydrogenase; GAPDH, glyceraldehyde-3-phosphate dehydrogenase. 


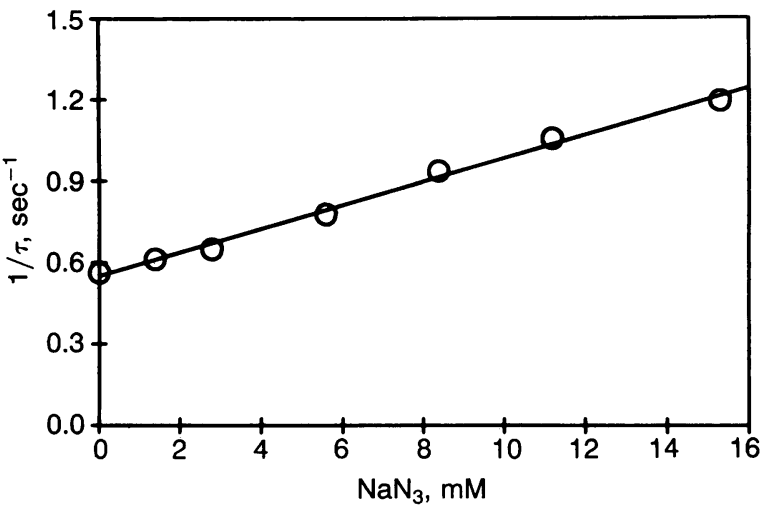

Fig. 1. Typical Stern-Volmer plot for quenching of alkaline phosphatase by azide. Data are plotted according to Eq. 1 to obtain the second-order quenching rate constant, $k_{\mathbf{q}}$, from the slope.

atoms and the solvent molecules were determined, and the closest distance found was taken as the distance of tryptophan to the protein surface.

\section{RESULTS}

Protein Quenching. Ten proteins with lifetimes ranging from $5 \mathrm{msec}$ to $1.6 \mathrm{sec}$ were chosen for the present study. Lifetimes for room-temperature tryptophan phosphorescence so far reported fall between $20 \mu \mathrm{sec}$ for free tryptophan in solution (10) and $>1 \mathrm{sec}$ for the most protected protein tryptophans. Therefore, one can expect the several tryptophan residues in any given protein to have quite different lifetimes. The phosphorescence decay observed nevertheless typically appears to be a single exponential in the proteins we have studied because the lifetime experiment easily focuses on the longest lived tryptophan.

Phosphorescence lifetimes were measured (by analysis for exponential decay) in the presence of increasing concentrations of four quite disparate quenchers: nitrite, azide, ethanethiol, and nicotinamide. The effect of added quenchers was monitored by decrease in the lifetime, and the secondorder quenching rate constant, $k_{\mathrm{q}}$, was obtained from the Stern-Volmer plot (11), as shown in Fig. 1, based on Eq. 1 (modified for lifetimes).

$$
1 / \tau=k_{\mathrm{q}}[\mathrm{Q}]+1 / \tau_{\mathrm{o}} .
$$

Here $\tau_{\mathrm{o}}$ is the lifetime in the absence of quencher $\mathrm{Q}$, and $\tau$ is the lifetime in the presence of the added quencher at concentration [Q].

Results are listed in Table 1. The proteins cover almost 3 orders of magnitude in lifetime and $>5$ orders of magnitude in sensitivity to quenching. For any given protein, however, the different quenchers have similar effects, evidently determined by some specific protein property. Further work was directed at identifying the structural determinants of tryptophan quenchability.

Identification of the Phosphorescent Tryptophans. To search for the structural correlates that determine tryptophan quenchability, it is necessary first to identify the tryptophan in each protein responsible for the measured phosphorescence-namely, the longest lived tryptophan.

Among the 10 proteins used here, 4 have but a single tryptophan. Trp-59 of RNase T1 has a 14-msec lifetime, occurs on a $\beta$-strand in a hydrophobic region against an $\alpha$ helix and a $\beta$-sheet (12), and is $2 \AA$ from the nearest surface water molecule. Trp-140 of staphylococcal nuclease has a 9-msec lifetime and also occurs close to the surface ( $2 \AA$ ) but is shielded from solvent contact by a hydrogen-bonding network (13). Trp-48 of azurin (from Pseudomonas aeruginosa) occurs within a $\beta$-barrel at the protein center (14), is 10 $\AA$ from the surface, and has a 400-msec lifetime. The position of Trp-109 in cod parvalbumin was judged by the analogous structure of the carp protein (15); it is at the $\mathrm{N}$-terminal region close to the surface $(2 \AA)$ and has a 5-msec lifetime.

For two of the multitryptophan proteins we studied, the phosphorescent tryptophan has been identified by other workers. Trp-314 of the dimeric LADH was identified as the long-lived phosphorescent tryptophan of LADH by Saviotti and Galley (1). It is in a $\beta$-sheet in the coenzyme-binding domain (16), has a 300 -msec lifetime, and is $5 \AA$ from the nearest solvent water molecule. Trp-310 of the GAPDH tetramer, identified as the phosphorescent tryptophan (17, 18 ), is $7 \AA$ from the aqueous surface and has a $750-\mathrm{msec}$ lifetime in our hands [Strambini and Gabellieri (17) report 395 $\mathrm{msec}]$. We worked with the porcine enzyme and utilized the $x$-ray structure of the homologous lobster protein $(19,20)$.

In the remaining four proteins, a choice for the most likely longest lived tryptophan had to be made from examination of the structure. Earlier work suggests that long-lived tryptophan phosphorescence relates to local structural rigidity. Strambini and Gonnelli (21) showed that the phosphorescence lifetime of indole derivatives free in solution increases dramatically as the viscosity is raised by added glycerol. Where long-lived phosphorescence has been assigned to a particular tryptophan, these tryptophans have been found to be in structured regions of the protein (reviewed in ref. 22). Therefore, we chose the most likely long-lived tryptophan on the basis of apparent local structural rigidity, as judged from its surrounding secondary structure.

Table 1. Protein phosphorescence-quenching constants for small molecules

\begin{tabular}{|c|c|c|c|c|c|c|}
\hline \multirow[b]{2}{*}{ Protein } & \multirow[b]{2}{*}{$\tau_{\mathrm{o}}, \sec$} & \multirow[b]{2}{*}{$\mathbf{r}, \AA$} & \multicolumn{4}{|c|}{$k_{\mathrm{q}}, \mathrm{M}^{-1} \cdot \mathrm{s}^{-1}$} \\
\hline & & & Nitrite & Azide & Ethanethiol & Nicotinamide \\
\hline \multicolumn{7}{|l|}{ Alkaline } \\
\hline phosphatase & 1.6 & 15 & 50 & 43 & 10 & 1 \\
\hline Azurin & 0.4 & 10 & $7.4 \times 10^{2}$ & - & $1.4 \times 10^{2}$ & $3.1 \times 10^{2}$ \\
\hline Aldolase & 0.04 & 8.5 & $5.7 \times 10^{3}$ & - & $5.7 \times 10^{2}$ & $1.6 \times 10^{3}$ \\
\hline Pronase & 0.7 & 7.5 & $2.5 \times 10^{3}$ & $1 \times 10^{4}$ & $5.2 \times 10^{2}$ & 18 \\
\hline GAPDH & 0.75 & 7 & $5.5 \times 10^{4}$ & $4.1 \times 10^{4}$ & - & $4 \times 10^{3}$ \\
\hline LADH & 0.3 & 5 & $4.7 \times 10^{4}$ & $1.1 \times 10^{5}$ & $4.2 \times 10^{4}$ & $4.6 \times 10^{4}$ \\
\hline RNase T1 & 0.014 & 2 & $5 \times 10^{5}$ & - & $2.0 \times 10^{4}$ & $3.2 \times 10^{4}$ \\
\hline Nuclease & 0.009 & 2 & $6.4 \times 10^{5}$ & $1.6 \times 10^{5}$ & $9.8 \times 10^{6}$ & - \\
\hline Parvalbumin & 0.005 & 2 & $2.7 \times 10^{6}$ & - & $3.1 \times 10^{6}$ & $3.1 \times 10^{5}$ \\
\hline Thermolysin & 0.01 & $\approx 1$ & $6.3 \times 10^{7}$ & - & - & - \\
\hline
\end{tabular}

For each protein, the Table lists: $\tau_{\mathrm{o}}$, the phosphorescence lifetime in seconds, measured at room temperature in solution in the absence of quencher; $r$, the shortest distance in angstroms between any indole atom of the phosphorescent tryptophan and a solvent water molecule; and $k_{\mathrm{q}}$, the measured second-order quenching rate constant in $\mathrm{M}^{-1} \cdot \mathrm{sec}^{-1}$. Data are from Calhoun et al. (7) or were determined in this work. 


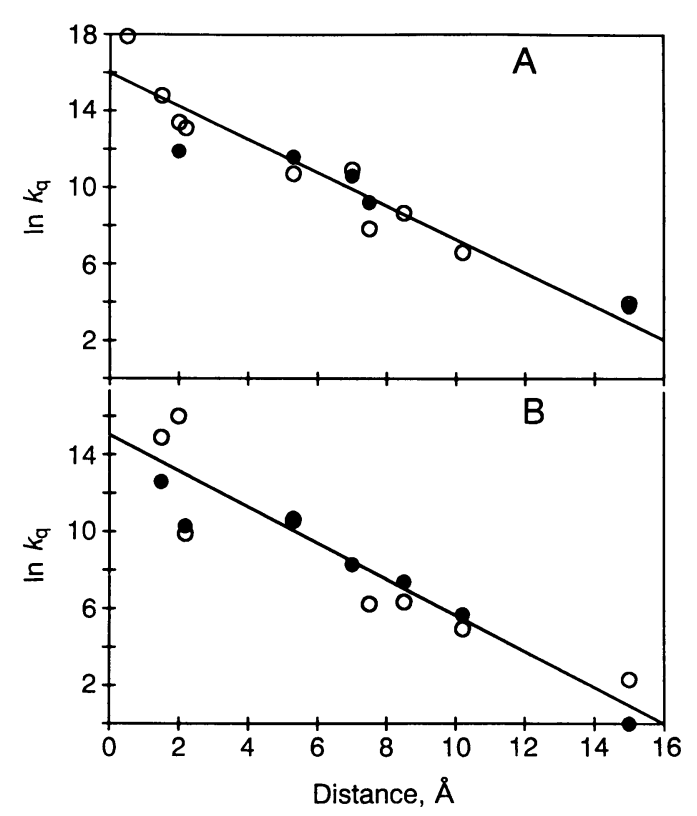

FIG. 2. Relationship between Stern-Volmer quenching constants and distance from the surface for the proteins in Table $1 .(A)$ Quenching constants for nitrite $(O)$ and azide $(\bullet)$. (B) Quenching constants for ethanethiol $(O)$ and nicotinamide $(\bullet)$. (The pronasenicotinamide datum point is omitted.)

Alkaline phosphatase, with the longest room-temperature phosphorescence lifetime yet observed, $\approx 1.6 \sec (1,4-7)$, is composed of two identical subunits, each containing three tryptophans. Trp-109, on an $\alpha$-helix connected to an extensive $\beta$-sheet region and deeply buried $15 \AA$ from the surface (23), is the apparent long-lived emitter. Rabbit skeletal muscle aldolase has four identical subunits, each containing three tryptophans (24). Trp-291 is in a loop close to the surface, Trp-311 is in an $\alpha$-helix, and Trp-147 is deeply buried in a $\beta$-barrel (25). Trp-147, expected to be the long-lived emitter, is $\approx 8.5 \AA$ from the surface. The Pronase preparation used is a mixture of two closely related enzymes, protease type A and type B, and contains a larger proportion of type B and some protein fragments (Sigma, personal communication). The type A protease has one tryptophan (Trp-66), and type B has two-Trp-67 turned out toward the solvent and Trp-66, the indole of which turns inward (26). The measured phosphorescence lifetime of $700 \mathrm{msec}$ is expected to be due to Trp-66, which is $\approx 7.5 \AA$ from the surface. Thermolysin has three tryptophans and exhibits a double exponential phosphorescence decay curve with lifetimes of $10 \mathrm{msec}$ and $\approx 50$ msec. The longer component was too weak to be measured accurately by our equipment. Trp- 55 and Trp- 115 , located in a $\beta$-structure near the $\mathrm{N}$-terminal region (27), are the most likely long-lived species. Both are near the surface and may be partially exposed. We estimate the distance at $\approx 1 \AA$.

Sensitivity to Quenching Depends on Distance from the Surface. In screening various possible correlates between structure and quenchability, the relationship shown in Fig. 2 was found. Fig. $2 A$ and $B$ plot the natural logarithm of the measured second-order quenching rate constant, $\ln k_{\mathrm{q}}$, against the distance of each tryptophan from the protein surface. When the distance to the surface exceeds a few angstroms, the curve assumes the form $k_{\mathrm{q}}=A \exp (-r / \rho)$.

The correlation observed in Fig. 2 includes all of the proteins and quenchers tested and holds $>5$ orders of magnitude in quenching constant. It appears that the curve for quenching by ethanethiol and nicotinamide (Fig. $2 B$ ) is slightly but significantly lower, or left-shifted, compared with the curve for nitrite and azide (Fig. 2A). This may be because of the larger size of the former quenchers, which can make their effective distance somewhat greater than the distance to the nearest solvent water plotted in Fig. 2. Scatter is relatively high for the tryptophans within a few angstroms of the protein surface, perhaps because of the roughness of the protein surface at the atomic level (28) possibly interacting with quencher shape and orientation. Also distances here are essentially at van der Waals contact distance and are somewhat ill defined.

\section{THEORY}

We wish to understand the relationship between the quenching rate constant and the donor-acceptor distance exhibited in Fig. 2. Here we consider the independence of rate on diffusion and the variation of rate with spatial separation when the quencher is excluded from close approach to the target tryptophan by the protein matrix.

The Rapid-Diffusion Limit. When donor-quencher interaction is efficient, so that nearly every collision produces a quenching reaction, the time dependence of the process is determined by the rate at which quencher molecules diffuse close enough to the donor for the interaction to occur $(29,30)$. Diffusion-limited reaction is often observed experimentally and holds, for example, for the quenchers used here when directed against the tryptophan analog $N$-acetyl-tryptophanamide in free solution (7).

Under other circumstances, the quenching reaction may be slow in comparison with encounter rates-for example, when quenching is constrained to occur through an inefficient mechanism such as long-range energy transfer. Here, diffusion-dependent spatial effects average out, the emitter "sees" the time-averaged quencher concentration, and the quenching rate becomes independent of diffusion. This rapid diffusion limit was demonstrated by Thomas et al. (31) and was found also by Calhoun et al. (4) in experiments on fluorescence quenching by resonance energy transfer. Similarly, the long-lived phosphorescent tryptophans studied here are quenched by external agents in a viscosity-independent, and therefore diffusion-independent, manner (7). (A mechanism involving rate-limiting diffusion inside the protein is ruled out by the observation of similar quenching by quenchers of very different molecular size and polarity.) Quenching of the excited-state tryptophan, then, depends upon the time-averaged concentration of quencher molecules external to the protein.

In the rapid-diffusion limit, the first-order quenching rate, $\phi$, is a summation of contributions from all quencher molecules in the system, each weighted according to the distance dependence of the quenching process, as in Eq. 2.

$$
\phi=\int_{\mathrm{V}} k(\mathbf{r}, t) \mathrm{Q}(\mathbf{r}, t) d^{3} r=10^{-3} N[\mathrm{Q}] \int_{\mathrm{V}} k(r) d^{3} r .
$$

Here $k(\mathbf{r}, t)$ is the first-order rate constant for interaction of a single donor-acceptor pair separated by the vector $r$. The quencher concentration $\mathrm{Q}(\mathbf{r}, t)$ in molecules per $\mathrm{cm}^{3}$ is zero within the protein and $10^{-3} N[\mathrm{Q}]$ over the external volume. $N$ is Avogadro's number, and [Q] is in mol/liter.

The quenching of excited states by long-range transfer can occur through a dipolar or an electron-exchange mechanism (32). For the present study, a dipolar resonant-energytransfer mechanism can be eliminated, since it requires donor-acceptor spectral overlap. In electron exchange, the transfer rate is proportional to the overlap of the exponential "tails" of the donor and acceptor wave functions and has the form

$$
k(\mathbf{r})=k_{0} \exp (-r / \rho)
$$


Here $k_{\mathrm{o}}$ is the first-order quenching rate when donor and quencher are in van der Waals contact, and $\rho$ is the incremental distance between donor and acceptor for a $1 / e$ attenuation of the transfer rate (33). For separations $r$ that are large in comparison with $\rho$, we make the approximation that $r$ is measured center-to-center and then substitute Eq. 3 into Eq. 2 to obtain the second-order rate constant, $\phi / Q$ :

$$
k_{\mathrm{q}}=\phi / \mathrm{Q}=10^{-3} N k_{\mathrm{o}} \int_{\mathrm{V}} \exp (-r / \rho) d^{3} r
$$

Protein Models. To obtain $k_{\mathrm{q}}$ for any given protein, the integral of Eq. 4 can be evaluated by numerical methods over all solvent space outside of the protein by using the protein's $\mathrm{x}$-ray coordinates. For certain model cases with simple geometrical shape, the integration can be performed to yield results in closed form. Pertinent examples are the spherically symmetric case ( $\left.k_{\mathrm{q}}^{\text {sph }}\right)$ with the tryptophan at the center and the planar case $\left(k_{\mathrm{q}}^{\mathrm{pl}}\right)$ with the tryptophan buried at a fixed distance below a protein surface having effectively infinite radius (Fig. 3). The distance of closest approach we denote by $a$.

The spherical protein of radius $a$ with a tryptophan at its center leads to Eq. 5.

$$
\begin{aligned}
k_{\mathrm{q}}^{\mathrm{sph}} & =4 \pi 10^{-3} N k_{\mathrm{o}} \int_{a}^{\infty} r^{2} \exp (-r / \rho) d r \\
& =4 \pi 10^{-3} N k_{\mathrm{o}}\left[a^{2} \rho+2 a \rho^{2}+2 \rho^{3}\right] \exp (-a / \rho) \\
\partial \ln \left(k_{\mathrm{q}}^{\mathrm{sph}}\right) / \partial a & =-(1 / \rho)\left[a^{2} /\left(a^{2}+2 a \rho+\rho^{2}\right)\right] .
\end{aligned}
$$

For the protein model with an effectively planar surface, Eq. 6 is obtained.

$$
\begin{aligned}
k_{\mathrm{q}}^{\mathrm{pl}} & =2 \pi 10^{-3} N k_{\mathrm{o}} \int_{a}^{\infty} d z \int_{0}^{\infty} \exp \left[-\left(r^{2}+z^{2}\right)^{1 / 2} / \rho\right] r d r \\
& =2 \pi 10^{-3} N k_{\mathrm{o}}\left[a \rho^{2}+2 \rho^{3}\right] \exp (-a / \rho) \\
\partial \ln \left(k_{\mathrm{q}}^{\mathrm{pl}}\right) / \partial a & =-(1 / \rho)[(a+\rho) /(a+2 \rho)] .
\end{aligned}
$$

Finally, we consider a protein with a deeply involuted surface in which one pocket with effective area $A$ provides access of quencher molecules to the tryptophan donor (Eq. 7).

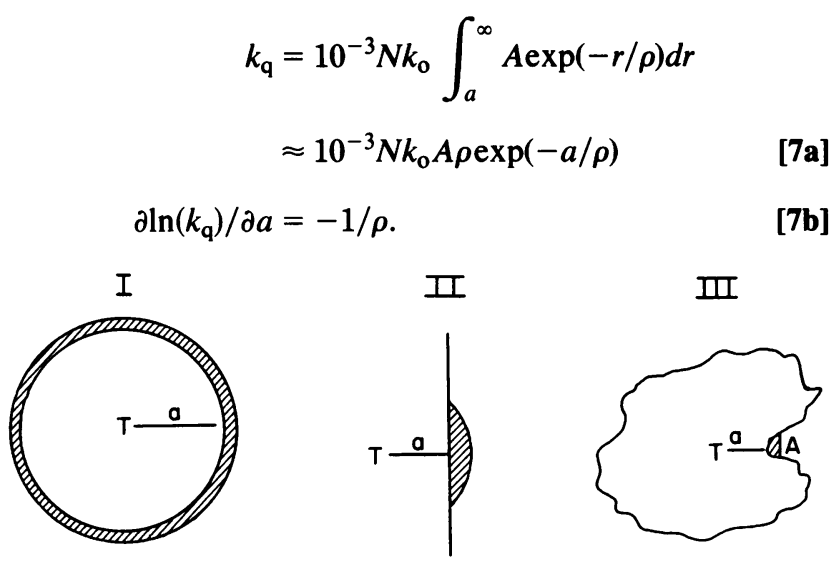

Fig. 3. Protein models with simple geometry. T represents tryptophan, and $a$ is the distance of closest approach to the aqueous surface. Schemes: I, tryptophan at the center of a spherical protein; II, tryptophan in a protein with planar surface; III, involuted protein surface. Shading shows the effective volume element (thickness $\rho$ ) occupied by the quencher.

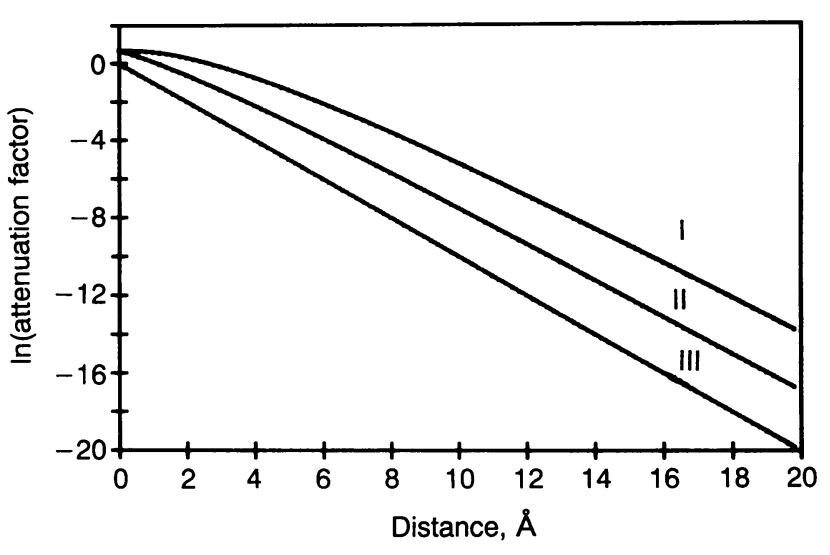

Fig. 4. Quenching probability (attenuation factor) for the spherical (I), planar (II), and involuted cases (III) plotted against the distance of closest approach. The curves are simulations of Eqs. 5, 6, 7 for the protein models with simple geometrical shape, with $\rho$ equal to 1 . In case I for the spherical model, the "attenuation factor" is defined as $\left[a^{2} \rho+2 a \rho^{2}+2 \rho^{3}\right] \exp (-a / \rho)$ (from Eq. 5a); the analogous factor is plotted for cases II and III.

Fig. 4 shows the dependence of these equations on $a$, with the natural logarithm of the "attenuation factor" plotted against $a$, the distance of closest approach. For these plots, $\rho$ was set equal to $1 \AA$, so that the limiting slope is normalized to -1 . For the spherical and planar protein models, the slope is flattened at short distances because of the pre-exponentialterms in Eqs. 5 and 6, and it approaches -1 at large distance, since the exponential term ultimately dominates.

\section{DISCUSSION}

Given the location of the phosphorescent tryptophans from crystallographic data, one can examine the effect of their burial on the rate of quenching by molecules in the solvent. Our data (Fig. 2) show that the quenching rate constant measured for many proteins decreases exponentially with the distance of the buried tryptophan from the protein surface. As shown by Eqs. 2-7 and the simulations in Fig. 4, this behavior is consistent with electron-exchange reactions between the buried tryptophans and quencher molecules that are excluded from the protein and in the rapid-diffusion limit.

Evaluation of $\rho$. Considerable work has been directed at measuring the distance dependence of electron-transfer reactions through proteins (e.g., refs. 33-37). Mayo et al. (34) emphasize the problem of establishing the exponential dependence of electron-transfer rate on distance through a protein environment. The results obtained here provide convincing evidence for this point and allow the distance parameter, $\rho$, to be evaluated from the slope of the plots in Fig. 2.

The best fit slopes for the experimental protein data in Fig. $2 A$ and $B$ are $0.87 \AA^{-1}$ and $0.90 \AA^{-1}$, respectively. The equations and simulations show that, in the range where the data are accurate $(\approx 5-15 \AA$ in Fig. 2$)$, the spherical, planar, and invaginated model proteins have apparent slopes -0.82 / $\rho,-0.91 / \rho$, and $-1.0 / \rho$, respectively. The slope produced by the invaginated model is equal to that characteristic of a monomolecular donor-acceptor pair; the slopes of the spherical and planar models are somewhat flatter. To obtain $\rho$ from the slope determined by the data for real proteins (Fig. 2), one wants to know the correct slope-flattening factor.

When the protein surface is convex in the immediate neighborhood of the point of closest approach, it can be inscribed between the spherical and planar model surfaces (when the point on the surface that marks the distance of closest approach to the buried tryptophan is taken as the common point of osculation of the three surfaces). The apparent slope in the region measured (Fig. 2) would then be 
less than $-1 / \rho$ by a factor between 0.82 and 0.91 , the factors pertinent for spherical and planar models. Similarly, when the general protein surface is concave about the point of closest approach, the surface can be inscribed between the planar and invaginated model cases, and the slope-flattening factor would be between 0.91 and 1.0. Since the process described by $k(r)$ (Eq. 3 et seq.) is exceedingly short range, quenching molecules near the point of closest approach can be expected to dominate the behavior. Thus, as the local protein surface diverges from sphericity at one extreme or from the invaginated shape at the other extreme, it quickly approaches the planar case, and we expect the average slope-flattening factor to be close to 0.91 . For the many proteins studied here, differences in surface shape about the point of closest approach may add to the variance observed in the data (Fig. 2), but this is overwhelmed by the 5 orders of magnitude in $k_{\mathrm{q}}$ covered by the values in Fig. 2. These considerations indicate that the slope measured in Fig. 2, $-0.89 \AA^{-1}$, should be approximated by the value $-0.91 / \rho$ and leads to a best estimate for $\rho$ of $\mathbf{1 . 0}$.

In electron-tunneling theory, the distance parameter $\rho$ is a sensitive function of the height of the energy barrier associated with tunneling through protein (38). This measure of how strongly forbidden it is for an electron to be found in the protein dictates the probability of transfer by tunneling. Our measurement of $1.0 \AA$ for $\rho$ can be compared with the prediction of Hopfield (38), who estimated $0.75 \AA$ for $\rho$, with greater values predicted when the donor is an excited-state wave function. A small amount of experimental data presently available for electron transfer from excited states in proteins, generally in reactions involving porphyrins, gives values for $\rho$ between 1.1 and $1.4 \AA$ [summarized by Mayo et al. (34)]. In those experiments, single donor-acceptor pairs at fixed distances were used, so that the transfer rate may be influenced by specific intervening residues. Our measurements use many proteins to determine $\rho$, so that the value obtained refers specifically to electron exchange when the intervening medium is represented by averaged protein matrix.

Evaluation of $\boldsymbol{k}_{\mathrm{o}}$. An extrapolation of the data for the rate constant $k_{\mathrm{q}}$ back to zero separation distance allows an estimation of the closest-contact unimolecular rate constant $k_{\mathrm{o}}$. The data in Fig. 2 point to an intercept value for $k_{\mathrm{q}} \approx e^{15.5}$ and thus estimate $k_{\mathrm{o}}$, the rate constant at van der Waals contact distance, at $\approx 10^{9} \mathrm{sec}^{-1}$ (using Eqs. 5a, 6a, or 7a with the parameter $A$ set at perhaps $10 \AA^{2}$ ). This value for $k_{\mathrm{o}}$ is in agreement with the results cited by Mayo et al. (34) and is much smaller than the value $10^{13} \mathrm{sec}^{-1}$ obtained from theoretical estimates for spin-allowed transitions. The value of $k_{\mathrm{o}}$ is likely to be influenced by the redox character of the reaction, geometrical factors, and the triplet nature of the donor wave function $(38,39)$.

This work was supported by research grants GM 21487 (to J.V.) and DK 11295 (to S.W.E.) from the National Institutes of Health and DCB-8718274 (to C.S.O.) from the National Science Foundation.

1. Saviotti, M. L. \& Galley, W. C. (1974) Proc. Natl. Acad. Sci. USA 71, 4154-4158.

2. Kai, Y. \& Imakubo, K. (1979) Photochem. Photobiol. 29, 261-265.

3. Vanderkooi, J. M., Calhoun, D. B. \& Englander, S. W. (1987) Science 236, 568-569.
4. Calhoun, D. B., Vanderkooi, J. M., Woodrow, G. V. \& Englander, S. W. (1983) Biochemistry 22, 1526-1532.

5. Calhoun, D. B., Vanderkooi, J. M. \& Englander, S. W. (1983) Biochemistry 22, 1533-1539.

6. Strambini, G. B. (1987) Biophys. J. 52, 23-28.

7. Calhoun, D. B., Englander, S. W., Wright, W. W. \& Vanderkooi, J. M. (1988) Biochemistry 27, 8466-8474.

8. Englander, S. W., Calhoun, D. B. \& Englander, J. J. (1987) Anal. Biochem. 161, 300-306.

9. Bernstein, F. C., Koetzle, T. F., Williams, G. J. B., Meyer, E. F., Jr., Brice, M. D., Rodgers, J. R., Kennard, O., Shimanouchi, T. \& Tasumi, M. (1977) J. Mol. Biol. 112, 535-542.

10. Eftink, M. R. \& Hagaman, K. A. (1986) Biophys. Chem. 25, 277-282.

11. Stern, O. \& Volmer, M. (1919) Phys. Z. 20, 183-188.

12. Heinemann, V. \& Saenger, W. (1982) Nature (London) 299, 27-31.

13. Cotton, F. A., Bier, C. J., Day, V. W., Hazen, E. E., Jr., \& Larsen, S. (1971) Cold Spring Harbor Symp. Quant. Biol. 36, 243-255.

14. Adman, E. T., Stenkamp, R. E., Sieker, L. C. \& Jensen, L. H. (1978) J. Mol. Biol. 123, 35-47.

15. Kretsinger, R. H. \& Nockolds, C. E. (1973) J. Biol. Chem. 248, 3313-3326.

16. Eklund, H., Nordstrom, B., Zeppezauer, E., Soderlund, G., Ohlsson, I., Boiwe, T., Soderberg, B. O., Tapia, O., Branden, C. I. \& Akeson, A. (1976) J. Mol. Biol. 102, 27-59.

17. Strambini, G. B. \& Gabellieri, E. (1989) Biochemistry 28, $160-166$.

18. Gabellieri, E. \& Strambini, G. B. (1989) Biophys. Chem. 33, 257-264.

19. Moras, D., Olsen, K. W., Sabesan, M. N., Buehner, M., Ford, G. C. \& Rossmann, M. G. (1975) J. Biol. Chem. 250, 91379162.

20. Harris, J. I. \& Waters, M. (1976) Enzymes 13, 1-49.

21. Strambini, G. B. \& Gonnelli, M. (1985) Chem. Phys. Lett. 115, 196-200.

22. Papp, S. \& Vanderkooi, J. M. (1989) Photochem. Photobiol. 49, 775-784.

23. Sowadski, J. M., Handschumacher, M. D., Murthy, H. M. K., Foster, B. A. \& Wyckoff, H. W. (1985) J. Mol. Biol. 186, 417-433.

24. Lai, C. Y., Nakai, N. \& Chang, D. (1974) Science 183, 12041206.

25. Sygusch, J., Beaudry, D. \& Allaire, M. (1987) Proc. Natl. Acad. Sci. USA 84, 7846-7850.

26. Delbaere, L. T. J., Hutcheon, W. L. B., James, M. N. G. \& Thiessen, W. E. (1975) Nature (London) 257, 758-763.

27. Colman, P. M., Jansonius, J. N. \& Matthews, B. W. (1972) J. Mol. Biol. 70, 701-724.

28. Richards, F. M. (1977) Annu. Rev. Biophys. 6, 151-176.

29. Smoluchowski, M. (1918) Z. Physik. Chem. (Leipzig) 92, 129168.

30. Collins, F. C. \& Kimball, G. E. (1949) J. Colloid Sci. 4, 425-437.

31. Thomas, D. D., Carlsen, W. F. \& Stryer, L. (1978) Proc. Natl. Acad. Sci. USA 75, 5746-5750.

32. Turro, N. J. Modern Molecular Photochemistry, ed. (Benjamin/Cummings, Menlo Park, CA), pp. 296-361.

33. Dexter, D. L. (1953) J. Chem. Phys. 21, 836-850.

34. Mayo, S. L., Ellis, W. R., Jr., Crutchley, R. J. \& Gray, H. B. (1986) Science 233, 948-952.

35. McLendon, G. (1988) Acc. Chem. Res. 21, 160-167.

36. Bechtold, R., Kuehn, C., Lepre, C. \& Isied, S. S. (1986) Nature (London) 322, 286-288.

37. Natan, M. J. \& Hoffman, B. M. (1989) J. Am. Chem. Soc. 111, 6468-6470.

38. Hopfield, J. J. (1974) Proc Natl. Acad. Sci. USA 71, 36403644.

39. Marcus, R. A. (1956) J. Chem. Phys. 24, 966-978. 